\title{
Tooth Fusion in Primary Teeth: A Case Series and Literature Review
}

\author{
Mohd Zulfadli Harun' Dayang Fadzlina Abang Ibrahim Siti Hajar Hamzah² Alaa Sabah Hussein² \\ ${ }^{1}$ Faculty of Dentistry, Universiti Teknologi MARA, Sg. Buloh Campus, \\ Malaysi \\ ${ }^{2}$ Centre of Paediatric Dentistry and Orthodontics Studies, Faculty of \\ Dentistry, Universiti Teknologi MARA, Sg. Buloh Campus, Malaysia \\ Address for correspondence: Alaa Sabah Hussein, Centre of \\ Paediatric Dentistry and Orthodontics Studies, Faculty of Dentistry, \\ Universiti Teknologi MARA, Selangor, Malaysia \\ E-mail:alaa@uitm.edu.my,dr_alaasabah@yahoo.com
}

\begin{abstract}
Tooth fusion is defined as the developmental dental anomaly in which two different tooth buds are fused into one during the development stage. It can involve both primary and permanent teeth, but its incidence has been found to be higher in anterior primary teeth involving mandibular anterior teeth. The etiology is unclear and may be related to physical pressure contact to the germ and genetic predisposition. The treatment options can vary in each child depending on the tooth mobility, morphology of the root canal system, the clinical appearance of the fused teeth, and the presence of malocclusion. This article presents three case reports of dental fusion between primary lateral incisor with primary canine in three patients. More interesting, Case 2 and case 3 were relatives and having similar fusion teeth. Clinical and radiographic examinations demonstrated the presence of incomplete fusion between primary lower right lateral incisor and canine in all cases, with bilateral aplasia of the succedaneous lateral incisor in case 1, whereas a unilateral aplasia of the correspondent permanent lateral incisor was noted in case 2 and 3. A preventive approach was provided, a fissure sealant on the deep fissure between the incomplete fusion teeth was placed in all cases, and periodic reviews were scheduled to prevent possible caries formation. Early detection of this anomaly ensures timely and appropriate clinical interventions to be performed, leading to treatment with predictable clinical outcomes and long-term prognosis and to prevent future complications. Furthermore, the incidence of fusion teeth that occurred in the similar region, similar teeth with positive family history indicates supporting the evidence of the shared genetic control of dental developmental disturbances.
\end{abstract}

Keywords: Dental anomalies, fusion, hypodontia, primary teeth

\section{Introduction}

Variations or anomalies in the number, size, and shape of the teeth can occur in both primary and permanent dentition.[1] One of the dental anomalies is tooth fusion that is defined as the combination between dentin and enamel of two or more separate developing teeth during intraosseous development.[2,3] Depending on the time of the fusion occurred, the teeth unite by the crown, pulp chamber or root and it can be complete (total) or incomplete (partial).[3] The crown is clinically bulky without any significant groove in a complete fusion with a normal looking pulp chamber. In an incomplete fusion, the separating groove is visible, and the pulp chamber can appear bifid or separated.[1]

The prevalence of fused teeth in primary dentition is $0.5 \%$ to $1 \%$ compared to $0.01 \%$ to $0.2 \%$ in permanent dentition, with primary lower incisors are the most fre-

How to cite this article: Harun MZ, Ibrahim DFA, Hamzah SH, Hussein AS. Tooth Fusion in Primary Teeth: A Case Series and Literature Review. J Pediatr Dent 2021;7(2):75-82 
quently affected.[2,4] It happens more often in the mandible's anterior region than the maxilla, and it can be bilateral or unilateral of jaw.[5] (1). Fusion can occur between teeth of the same dentition, mixed dentitions or even between normal and supernumerary teeth.[6] No significant differences between males and females were reported in the literature[7] whereas racial differences have been reported indicating a higher incidence among the Japanese and Chinese population compared to the Caucasian population.[3]

Although the etiological factors of the fused teeth are not fully understood, some theories have been raised to address the issues. The first theory explained a possible physical pressure or force generated such as trauma during growth and development of tooth could induce a close contact of the tooth to the germs, which may produce a necrosis of the epithelial tissue that separates them and result in the fusion. [8] The other theory suggested the use of thalidomide or the occurrence of viral infection during pregnancy.[9] Genetics also may play a vital role in the formation of this anomaly. Fusion teeth have been reported to be associated with cleft lip and in X-linked congenital abnormalities. $[10,11]$ Fusion teeth also noted with some dental anomalies such as supernumerary teeth, hypodontia, peg-shaped incisors, conical teeth, macrodontia, dens in dente, and some non-dental anomalies like nail disorders and syndactyly.[7,8]

Since this anomaly clinically needs demanding treatment, a comprehensive evaluation and examination, including radiography, is critical. A thorough radiographic evaluation is a the most prior to therapy for a favorable result. Fused teeth may share an enlarged pulp chamber and a single root canal, may have separate root canals or bifurcated pulp chambers[12,13] The pulp chamber in case of an incomplete fusion can appear bifid or separated.[7] Moreover, several studies revealed inadvertently findings anomaly of succeeding permanent teeth during radiographic investigation. $[10,14]$ Hence, early detection and diagnosis of fusion in primary teeth is imperative to avoid clinical problems in the future such as dental caries, delay exfoliation of primary teeth, unerupted or impaction of the subsequent permanent teeth, permanent tooth agenesis, presence of supernumerary teeth, presence of permanent fusion teeth, and crowding due to the lack of space. [2,3]

The treatment options for this type of anomaly should consider the intraoral condition, oral hygiene, and occlusion. It could be an extraction in cases of impaction and delay eruption of the permanent succes- sor, pulp therapy when the pulp is compromised, orthodontic treatment for the lack of space and crowding, separation tooth when the fusion only occurs in the crown, and esthetic treatment if there are complaints on the part of the patient. [3,6] A preventive approach as part of the treatment plan to protect the fused primary tooth can be suggested. This includes topical fluoride application, dietary changes, and regular follow-up.[3] This paper highlights a clinical case series of a fusion between a primary lower lateral incisor and canine associated with a congenital missing of the corresponding permanent lateral incisor along with a discussion on the treatment plan in uncooperative children. Additionally, it depicts tooth fusion occurrence in the cousins contributing evidence to an understanding of genetically controlled dental anomaly patterns. To the best of our knowledge, this is the first case reported in the literature about the incidence of dental fusion among the relatives.

\section{Case Presentation}

\section{Case 1}

A six-year-old Malay boy reported to Pediatric Dentistry Specialist Clinic at Universiti Teknologi MARA accompanied by his mother with a complaint of multiple tooth cavities. The medical history of the child was non-contributory and there was no history of dental trauma. The family history did not reveal any dental abnormality. His older sister who was treated at the same clinic and presented no dental anomalies. Likewise, this first dental visit showed negative extraoral examination. However, intraoral examination disclosed that the patient is in mixed dentition stage with the presence of multiple carious teeth including bilateral upper and lower primary molar, upper right lateral and central primary incisor, bilateral lower permanent first molar and retain root upper left primary central incisor. The other findings include fusion of the primary lower right lateral and lower right canine (Fig. 1a). The three anterior teeth, which are the permanent upper left central incisor, primary lower left central incisor, and primary lower lateral incisor was in a crossbite relationship (Fig. 1b). Bilateral posterior crossbite is also noted in this patient.

The child has no habit of brushing the teeth before bedtime and usually fall asleep while sucking the milk bottle. This has causes plaque accumulation in the upper and lower arches. The analysis of the orthopantomogram (OPG) and intraoral periapical (IOPA) radiographs led to the diagnosis of fusion teeth with the pri- 
mary lower right lateral incisor was in an incomplete fusion with the primary lower right canine in addition to the presence of two pulp chambers (Fig. 1c). Another finding was congenital missing of the permanent lower right and left lateral incisors (Fig. 1d). Few treatment options were considered for this case. However, due to poor oral hygiene, especially in the lower right area (Fig. 1e), a preventive approach was the best option to avoid dental problems such as dental caries.

The dental anomalies and the treatment plan were explained to the mother, and signed informed consent was obtained authorizing the treatment and to disclose and publish this case report. Dental prophylaxis and topical fluoride application were performed as well as oral hygiene instruction was given in the presence of his mother. Fissure sealant with resin composite was placed on the deep groove between the incomplete fusion teeth (Fig. 1f). Preventive resin restoration was performed on the bilateral lower first permanent molar. Glass ionomer cement restorations were performed on the upper and lower primary molars. This patient is under review every 3-month for oral hygiene monitoring and fluo-
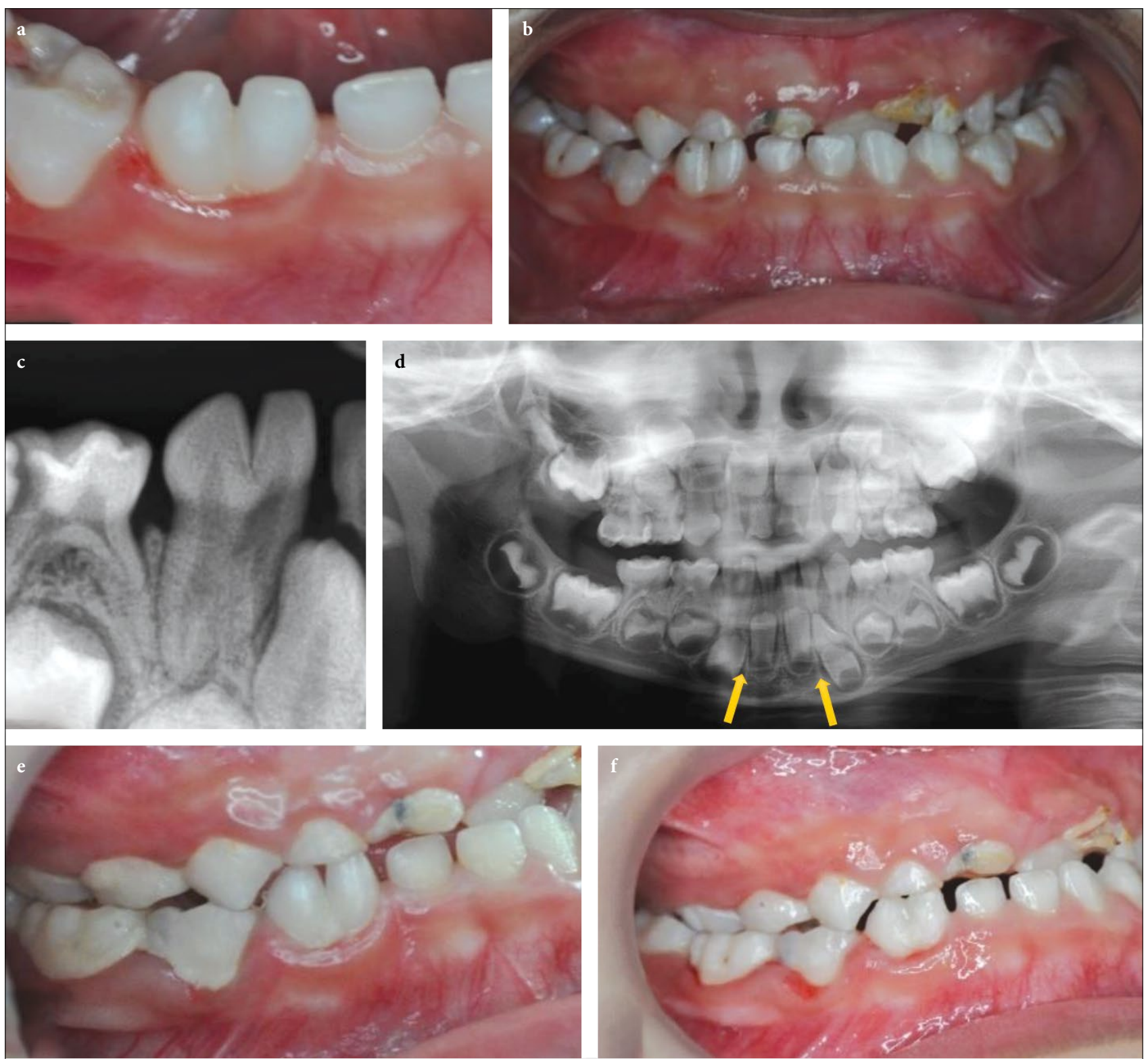

Figure 1. (Case1): Preoperative intra-oral photograph of incomplete fusion primary tooth (a) Anterior view photograph showing multiple caries with anterior and posterior crossbite (b) Periapical radiograph of fusion primary tooth showing two separate pulp chamber (c) Orthopantomogram showing a mixed dentition stage and noted missing lower right permanent lateral incisor tooth (d) Preoperative photograph showing uncomplete fusion primary tooth with plaque accumulation (e) Post-operative photograph showing oral prophylaxis and fissure sealant placed were done (f) 
ride application as he is at high risk for caries. The eruption of the permanent teeth also will be monitored.

\section{Case 2}

A four-year-old Malay girl was referred to Pediatric Dentistry Specialist Clinic at Universiti Teknologi MARA for the examination of double teeth. The medical history of the child was non-contributory. The family history did not reveal any dental abnormality and no consanguinity was reported in the parents. The child appeared healthy with no history of dental trauma.
Intraoral examination revealed enlarged bifid crown due to fusion of the primary lower right lateral and lower right canine, with a central depression along the entire labial and lingual vestibular surfaces that tended to divide the piece partially (Figs. 2a, b). No dental caries was clinically noted on other teeth. Her oral hygiene status was fair. The child's nephew (Case 3) presented with a similar condition and coincidently occurred at a similar location.

The IOPA radiograph of the region exhibited that the crowns and the roots were fused resulting in joined pulp canal with an incomplete union of their pulp
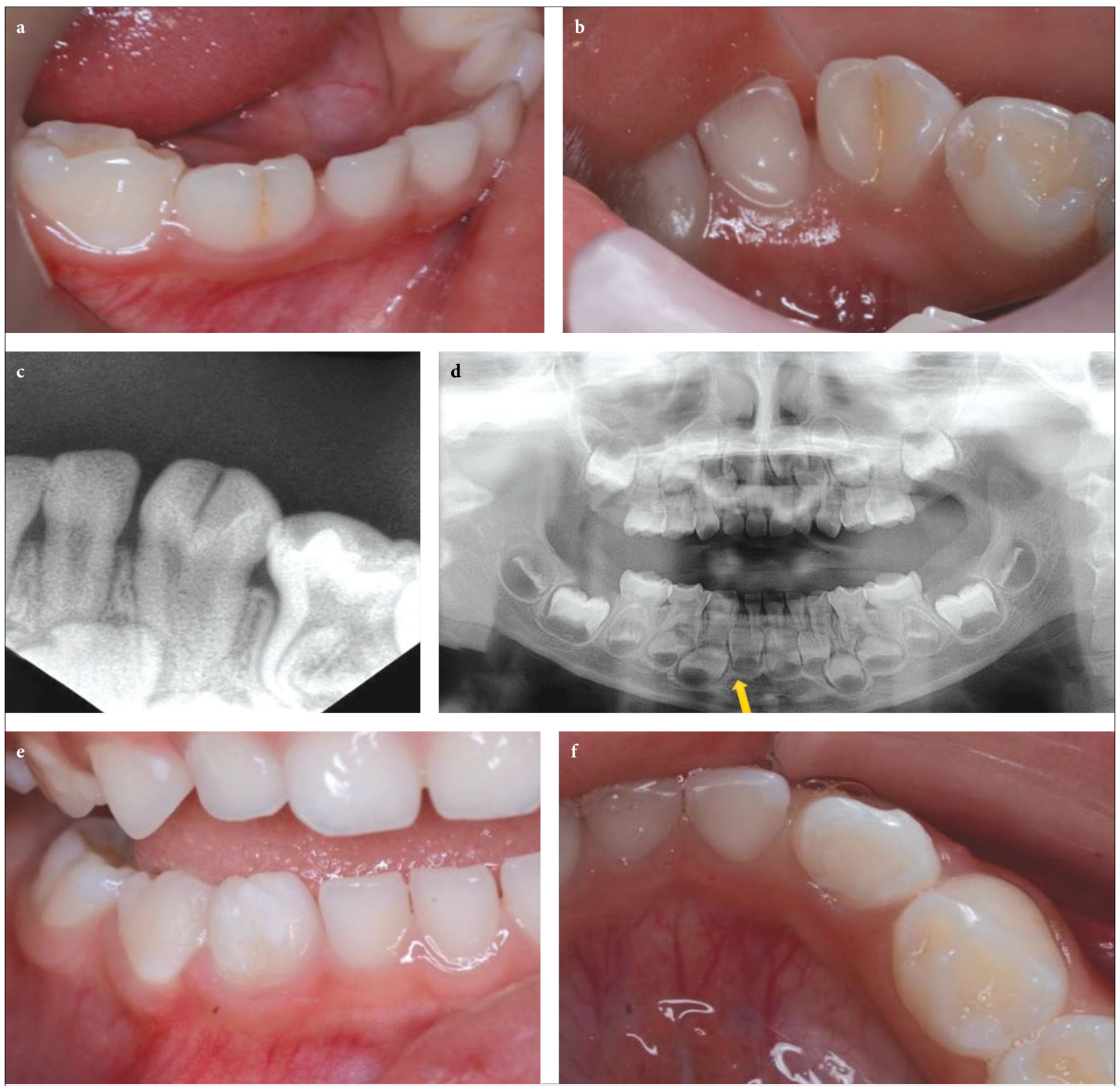

Figure 2. (Case2): Preoperative intra-oral photographs buccal (a) and lingual (b) view of fusion tooth lower right lateral and lower right canine. IOPA radiograph showing fused tooth lower right lateral and lower right canine (c) Orthopantomogram disclosing missing permanent right lateral incisor (d) Post-operative photographs of fusion tooth after fissure sealant application buccal view (e) and lingual view (f) 
chambers and the OPG revealed missing lateral successor tooth (Figs. 2c, d). The fused tooth had one big root and two pulp chambers. The dental anomalies were explained to the child and her mother. Written consent was obtained from the mother for the agreed dental treatment and the use of the records and photographs for publication purposes. Based on the clinical and radiological examinations treatment plan of fissure sealant was formulated. Fissure sealant with resin composite was placed on the deep groove between the incomplete fusion teeth (Figs. 2e, f) followed by topical fluoride application. Oral hygiene instruction was given in the presence of the parent and a regular follow-up was advised. Follow-up is expected to continue until the eruption of permanent teeth.

\section{Case 3}

A 5-year-old Malay slow learner boy was referred to Pediatric Dentistry Specialist Clinic at Universiti Teknologi MARA for the examination of fused teeth. The medical history of the child patient was non-contributory. The family history did not reveal any dental abnormality and no consanguinity was reported in the parents. The child appeared healthy with no history of dental trauma. The clinical extraoral examination did not show any different alteration. The clinical intraoral examination revealed the presence of fused teeth involving crowns of tooth lower right lateral and lower right canine (Figs. 3a, b). There was a deep grove on the labial and lingual surface with incisal notching. No dental caries was clinically noted on other teeth. His oral hygiene status was fair.

The patient was uncooperative during IOPA radiograph procedure. The OPG was taken and revealed the presence of fused tooth with two independent and converging roots with a single pulp chamber (Fig. 3c). Another finding was congenital missing of the permanent lower right lateral incisors. Parents were informed about fused teeth and the missing corresponding permanent tooth buds. Based on the clinical and radiological examinations treatment plan of fissure sealant was formulated. Fissure sealant with resin composite was placed on the deep groove between the incomplete fusion teeth (Fig. 3d, e) followed by topical fluoride application. Oral hygiene instruction was given to the child in the presence of the parent and a regular followup was advised. Follow-up is expected to continue until the eruption of permanent teeth. Written consent was obtained from the mother for the agreed dental treatment and the use of the records and photographs for publication purposes.

\section{Discussion}

Since this fusion tooth anomaly is considered rare, a detailed patient history, clinical evaluation and radiographic examination are essential for a correct diagnosis to provide successful management of fused primary teeth in young children. This anomaly is strongly associated with anomalies in permanent dentition. The presence of a double primary tooth can also cause delayed in resorption of root due to greater root surface area relative to the size of permanent successor crown and may lead to delay or ectopic resorption of permanent successor.[15] Radiographs including intraoral periapical and panoramic are required to detect the morphology of the root and canal involve as well as the presence of its corresponding permanent tooth. $[10,14]$

Gemination is often confused with fused teeth. Gemination, by definition is an anomaly caused by a single tooth germ that attempted to divide during development, resulting in a bifid crown. Mader's "two tooth" rule can be used to differentiate between fusion and gemination. Any double tooth that is counted as one and less total number of teeth are present in the dental arch, a fusion is considered. However, when the double tooth is counted as one and the number of teeth in the dental arch is normal, it is termed as gemination or maybe a case of fusion between normal and supernumerary teeth. A diagnostic consideration should aware that supernumerary teeth clinically slightly peculiar or cone-shaped, therefore, fusion between normal anomalies of supernumerary teeth will show differences in two halves of the joined crown. However, in gemination, the two halves of the joined crown are mirror images and there is a buccolingual groove that extends to the incisal edge.[16] Furthermore, radiographically fused teeth present two separate pulp chambers with two root canals, whereas geminated teeth usually have a single root. [5,17] Based on the teeth number, crown morphology and the radiographic evaluation indicating a case of fusion and not germination in all three cases, because there was one tooth less than normal in the lower arch of the patients.

Dental fusion is classified into total or partial fusion based on the stage in which it happened. A total fusion occurs at the early stages of development before calcification begins, resulting in a normal sized or slightly bigger than normal tooth. If the fusion happens during the calcification stage, the fusion will be partial where the tooth has the size of two crowns fused together, a bifid crown, or both.[11,18] Consequently, our three cases can be considered an incomplete fusion since it is possible to differentiate where the fusion happened 


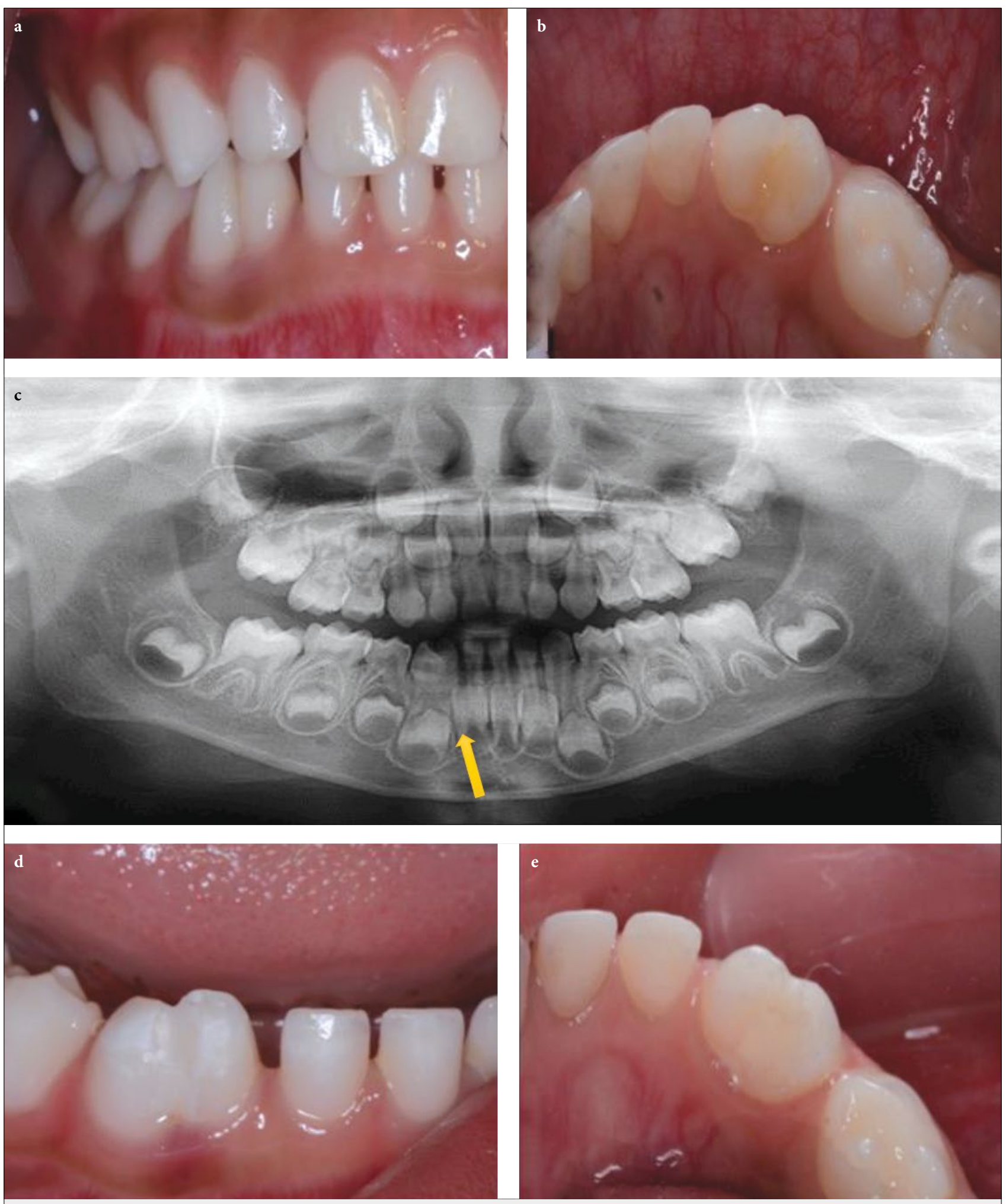

Figure 3. (Case 3): Preoperative intra-oral photographs buccal (a) and lingual (b) view of fusion tooth lower right lateral and lower right canine. Orthopantomogram showing fusion tooth lower right lateral and lower right canine and congenitally missing tooth right lower lateral incisor (c) Post-operative photographs of fusion tooth after fissure sealant application buccal view (d) and lingual view (e)

between the fused teeth. Two pulp chambers are clearly identified in the radiographs in case 1 and 2 whereas a single pulp chamber is recognized in case 3 .
The prevalence of dental fusion in primary dentition is higher in the lower arch particularly in the anterior region.[3] All three cases involved the man- 
dibular anterior teeth (primary lower right lateral and lower right canine) and were unilateral in which it is the same in most of the reported case involving primary teeth fusion of the lower anterior teeth of incisor teeth and usually occurs unilaterally.[9] Acikel et al in 2018 observed 50 primary fused teeth in 40 patients, with the most prevalent fusion was detected between the mandibular lateral and canine teeth. This condition is known to increase the possibility of permanent tooth anomalies.[14] Another study by Sekerci et al comprising of six primary fusion teeth cases revealed three cases with the absence of the lower permanent lateral incisor teeth.[19] Similarly, to our present reports, bilateral congenital missing lower permanent lateral incisor was noted in case 1 whereas unilateral agenesis of a permanent successor was identified in case 2 and 3 .

The etiology of this anomaly remains unknown; however, many researchers describe it as a combination of genetic and environmental factors. Fusion can occur in the developmental stages of dental morpho-differentiation. Pressure or physical force resulting in close contact between the two tooth germs is also reported to be a main possible cause of tooth fusion. $[9,10]$ No clear etiology was concluded for case 1 because there was no family history of tooth fusion or fetal alcoholic exposure. Hence, another etiological factors for instance viral infection during pregnancy or hypervitaminosis may contribute in the occurrence of the dental fusion. $[11,12]$ In case 2 and 3 who were relatives, we concluded the genetic factor was the main etiological factor for this anomaly since heredity seems to have an important implication in the development of this anomaly as evidenced in family.[1,2]

The treatment depends upon the patient prerequisite, the teeth involved, and the degree of involvement. Study by Shah et al in 2012 highlighted three additional aspects to consider before deciding on the best therapy for this dental anomaly is the prognosis of the tooth, the patient's cosmetic expectations, and any orthodontic therapy problems.[20] Based on the most recent systematic review performed by Bernardi et al[3], the management options for fused primary teeth comprise diverse treatment modalities ranging from no intervention, preventive measures, orthodontic treatment, restorative treatment or surgical measures such as crown modification with or without hemisection or extraction. Some studies suggested the application of preventive measures to the primary fused teeth. Sekerci et al suggested prophylactic filling of the deep grooves and fluoride application as a further preventive approach.[19] Deep groove between the fused teeth may lead to food and plaque impaction and be more susceptible to dental caries occurrence. Although the absence of caries on the fused teeth in all cases reported in this paper could lead to the underestimation of this tooth anomaly, complications involving the pulp and the occlusion can still occur. Therefore, accurate monitoring is one of the most recommended initial diagnostic measures. Considering the above points, fissure sealant was placed on the deep groove between the fused followed by full mouth topical fluoride application in all present cases.

Additionally, a complication that seems to be associated with the presence of fused primary teeth is the absence of permanent successor teeth. Although primary fusion teeth may be considered as harmless anomaly, their existence can result in excess dental space, occlusal disturbances, and delayed eruption of the permanent successors.[13] In this study, cases 2 and 3 presented primary fusion teeth that associated with the absence of the correspondent permanent lateral incisor. However, case 1 presented with a bilateral absence of permanent lateral incisors. Therefore, a good diagnosis usually consists of a radiographic assessment for the presence of the permanent successor teeth to establish an efficient therapy plan. The preventive or restorative treatment of the fused teeth is essential for a missing permanent successor tooth to facilitate prosthetic therapy in the future when the child reaches the appropriate age around 18 years. [3]

Fusion teeth usually entail a multidisciplinary approach for a better prognosis outcome. It is important to identify this anomaly early and to perform a detailed analysis for each case to formulate a conservative and individualized treatment plan. The poor oral hygiene condition of the children and the absence of a permanent tooth successor led to a preventive protocol as the best option to protect the fused tooth and to avoid any future complication. A 3-month review was scheduled to maintain his oral health and to reinforce positive oral hygiene practices and healthy habits for the patients, who had a high caries risk while allowing for the early management of potential dental complications that might occur in the future. Moreover, the periodic follow-up visits are expected to continue monitoring the eruption of the permanent central incisors and planning the definitive treatment for the congenital missing of permanent lateral incisor which will be required a multidisciplinary effort comprising pediatric dentist, orthodontist, radiologist, and prosthodontist in the future. 


\section{Conclusion}

Tooth fusion can be associated with a genetic tendency and agenesis of its permanent successor. Comprehensive clinical and radiographic evaluations are important to detect the dental developmental anomaly earlier. Diagnosis and management of fusion teeth in young children have always been challenging. The most adequate dental intervention is from a multidisciplinary approach involving a pediatric dentist, orthodontist, and prosthodontist considering the child's condition, expectation, and degree of cooperation with dental treatment. A follow-up visit is very fundamental for the fused tooth cases along with preventive procedures and close monitoring despite the absence of signs and symptoms. Furthermore, the incidence of fusion teeth that occurred in the similar region, similar teeth with positive family history indicates supporting the evidence of the shared genetic control of dental developmental disturbances.

Financial Disclosure: Nil.

Conflict of Interest: None declared.

\section{References}

1. Aguiló L, Gandia JL, Cibrian R, Catala M. Primary double teeth. A retrospective clinical study of their morphological characteristics and associated anomalies. Int J Paediatr Dent 1999;9(3):175183 doi:10.1046/j.1365-263x.1999.00131.x

2. Guimarães Cabral LA, Firoozmand LM, Dias Almeida J. Double teeth in primary dentition: report of two clinical cases. Med Oral Patol Oral Cir Bucal 2008;13(1):E77-E80

3. Bernardi S, Bianchi S, Bernardi G, et al. Clinical management of fusion in primary mandibular incisors: a systematic literature review. Acta Odontol Scand 2020;78(6):417-424 doi:10.1080/000 16357.2020.1734233

4. Zengin AZ, Celenk P, Gunduz K, Canger M. Primary double teeth and their effect on permanent successors. Eur J Paediatr Dent 2014;15(3):309-312

5. Song CK, Chang HS, Min KS. Endodontic management of supernumerary tooth fused with maxillary first molar by using conebeam computed tomography. J Endod 2010;36(11):1901-1904 doi:10.1016/j.joen.2010.08.026

6. Costa AC, Nascimento BS, Silva JLC, Santos AMC, Cruz SCC, Fernandes MLMF. Fused teeth in the primary dentition: clinical case report. RGO, Rev Gaúch Odontol 2020;68:e20200051
7. Hattab FN. Double talon cusps on supernumerary tooth fused to maxillary central incisor: Review of literature and report of case. J Clin Exp Dent 2014;6(4):e400-e407 doi:10.4317/jced.51428

8. Giffoni TCR, Brandt GZ, Rocha IS, Ramos AL, Provenzano MGA, Fracasso MLC. Relation of dental anomalies with occlusal alterations in the pediatric patients. Pesqui Bras Odontopediatria Clin Integr 2019;19(1):1-10

9. Kjaer I, Daugaard-Jensen J. Interrelation between fusions in the primary dentition and agenesis in the succedaneous permanent dentition seen from an embryological point of view. J Craniofac Genet Dev Biol 2000;20(4):193-197

10. Nik-Hussein NN, Abdul Majid Z. Dental anomalies in the primary dentition: distribution and correlation with the permanent dentition. J Clin Pediatr Dent 1996;21(1):15-19

11. Jahanimoghadam F. Dental anomalies: An update. Adv Hum Biol 2016;6:112-118 doi:10.4103/2321-8568.195316

12. Martinez F, Segura F. Dental Union Abnormalities: Literature Review. Acta Scientific Dental Sciences 2018;2(11):36-40

13. Aydinbelge M, Sekerci AE, Caliskan S, Gumus H, Sisman Y, Cantekin K. Clinical and radiographic evaluation of double teeth in primary dentition and associated anomalies in the permanent successors. Niger J Clin Pract 2017;20(7):847-851 doi:10.4103/1119-3077.183246

14. Acikel H, Ibis S, Sen Tunc E. Primary fused teeth and findings in permanent dentition. Med Princ Pract 2018;27(2):129-132 doi:10.1159/000487322

15. Duncan WK, Helpin ML. Bilateral fusion and gemination: a literature analysis and case report. Oral Surg Oral Med Oral Pathol 1987;64(1):82-87 doi:10.1016/0030-4220(87)90121-6

16. Mader CL. Fusion of teeth. J Am Dent Assoc 1979;98(1):62-64 doi:10.14219/jada.archive.1979.0037

17. Soghli N, Panjnoush M, Johari M. Incidental finding of a supernumerary tooth fused to a mandibular second molar using cone beam computed tomography (CBCT): A case report. Int J Sci Res Dent Med Sci 2020;2:20-22

18. Da Costa GC, Chalakkal P, De Souza N, Gavhane S. Bilateral complete and incomplete fusion of incisors and its management. Contemp Clin Dent 2017;8(1):171-174 doi:10.4103/ccd. ccd_1090_16

19. Sekerci AE, Sisman Y, Ertas ET, Gumus H, Ertas H. Clinical and radiographic evaluation and comparison of six cases of fusion involving the primary dentition. J Dent Child (Chic) 2012;79(1):34-39

20. Shah P, Chander JM, Noar J, Ashley PF. Management of 'double teeth' in children and adolescents. Int J Paediatr Dent 2012;22(6):419-426. doi:10.1111/j.1365-263X.2011.01209.x 